\title{
SMART CITY - UMA OPORTUNIDADE DE APLICAÇÃO DE PEQUENOS SISTEMAS GERADORES COM ENERGIA RENOVÁVEL*
}

\author{
Márcio Zamboti Fortes ${ }^{1}$ \\ Vitor Hugo Ferreira ${ }^{1}$ \\ Ivan de Souza Machado ${ }^{2}$ \\ Weules Fernandes Correia ${ }^{3}$
}

\section{Resumo}

A alguns anos os setores residencial e industrial tem se deparado com mudanças de paradigmas que tangem a necessidade de melhorias em suas atividades, com 0 intuito de desenvolver processos com menor impacto ambiental, sustentáveis e com eficiência energética, objetivando reduzir o consumo de energia elétrica. $\mathrm{O}$ uso de tecnologias baseadas em fontes renováveis para a geração de energia elétrica, tais como a solar e a eólica, tem apresentado-se como uma alternativa a ser implementada por estes setores para atender às exigências do mercado, se beneficiando da difusão da tecnologia e uso destes equipamentos, para a consciente utilização da energia elétrica. A instalação e avaliação de novas fontes de geração de eletricidade estão sendo desenvolvidas pelo projeto Cidade Inteligente de Búzios através da implantação de painéis fotovoltaicos e aerogeradores de pequeno porte em unidades consumidoras residenciais, comerciais e industriais e alguns resultados estão apresentados neste texto.

Palavras-chave: Geração de energia; Fontes alternativas; Cidades inteligentes.

\section{SMART CITY - AN OPPORTUNITY TO APPLY SMALL RENEWABLE ENERGY GENERATION SYSTEMS}

\begin{abstract}
For some years the residential and industrial sectors have come up against paradigm changes that concern the need for improvements in their activities, in order to develop processes with less environmental impact, sustainable and energy-efficient, aiming to reduce energy consumption. The use of technologies based on renewable sources for power generation, such as solar and wind, has emerged as an alternative to be implemented for these sectors to meet the requirements of the market, benefiting from the dissemination of technology and equipment for the conscientious use of electricity. The installation and evaluation of new sources of electricity generation are being developed by the Smart City Buzios Project through the deployment of photovoltaic panels and small wind turbines in residential, commercial and industrial consumer units and some results are presented.
\end{abstract}

Keywords: Energy generation; Alternative electric power sypply; Smart cities.

Doutorado em Eng. Elétrica, Prof. Adjunto, TEE, Universidade Federal Fluminense, Niterói, RJ, Brasil.

2 Mestrando em Eng. Elétrica e Telecomunicações, PPGEET, Universidade Federal Fluminense, Niterói, RJ, Brasil.

3 Eng. Eletricista, Gestor de P\&D, Ampla Energia e Serviços S.A., Universidade Federal Fluminense, Niterói, RJ, Brasil. 


\section{INTRODUÇÃO}

Diante de cenários que apontam para o aumento da demanda de energia elétrica, leis ambientais mais rígidas, busca pela sustentabilidade, e eficiência energética para redução do consumo de energia elétrica tendo em vista seu custo, a utilização de fontes renováveis de energia apresentam-se como alternativas para a diminuição do consumo de energia elétrica seja no setor residencial/comercial e industrial.

Entre as tecnologias de geração de energia que estão se destacando neste contexto estão a energia solar através da utilização de sistemas de painéis fotovoltaicos, e a eólica com aerogeradores de pequeno porte, quando se avaliam a inserção de novos sistemas geradores para suprir ou complementar o consumo de pequenos setores.

A conversão da luz solar diretamente em energia elétrica através do sistema fotovoltaico apresenta características relevantes quando comparada aos insumos tradicionais, pois esta possui uma fonte abundante, não emite poluentes em sua operação, não gera poluição sonora, e é composta por módulos que podem ser aplicados em diferentes atividades [1]. As células fotovoltaicas existentes no mercado são de silício monocristalino, policristalino e filme fino, sendo que as células de silício cristalino podem ser monocristalinas, policristalinas ou de Arseneto de gálio. As de filme fino podem ser de silício amorfo, telureto de cádmio, sulfureto de cádmio e cobre-índio-gálio-selênio.

Os aerogeradores são equipamentos que através da energia cinética dos ventos produzem energia mecânica movendo o eixo do rotor que é acoplado a um gerador que converte a energia de movimento em energia elétrica. Os aerogeradores são classificados de acordo com a posição de seu eixo, sendo denominados aerogeradores de eixo vertical e horizontal.

O projeto Cidade Inteligente de Búzios tem como um de seus objetivos difundir as novas tecnologias de geração para o desenvolvimento da utilização destas no setor residencial, serviços públicos, comercial e industrial, produzindo resultados que contribuirão para estudos da inserção destes equipamentos na rede de distribuição.

A análise da aplicação destes equipamentos por parte dos investidores no setor industrial é realizada de maneira mais detalhada e cautelosa, pois se trata de uma decisão que pode afetar o futuro do empreendimento, posto que, são ponderados riscos dos custos esperados, a disponibilidade da fonte, a confiabilidade do fornecimento, o desenvolvimento em que a tecnologia se encontra, e outros fatores tais como, produção sustentável, legislação governamental, questões ambientais, e regulamentações [2]. Além destes fatores, o setor industrial quando comparado ao residencial pode se beneficiar de espaços não utilizados como telhados de galpões, áreas de armazenamento, estações de tratamento de água e esgoto entre outros, devido, em geral, à sua considerável aérea de ocupação espacial.

A utilização de sistemas de geração fotovoltaica e solar térmica podem apresentarse como uma forma de introduzir um caráter sustentável a determinados processos, sendo demonstrado interesse pela indústria de petróleo e gás para algumas aplicações garantindo a redução dos impactos ambientais [3]. Como exemplo, podese citar a proposta de aplicação de um sistema fotovoltaico na implantação na unidade CEVITAL, localizada na Larbaa em Algies, para obtenção de hidrogênio a partir da eletrólise da água [4].

De acordo com a empresa o projeto aplicado pode ser implantado como um sistema híbrido para geração de energia elétrica e térmica por meio da energia solar, proporcionando melhoras energéticas devido ao aumento global da produção de 
energia dentro do processo, considerando a localização da geração solar e o sistema à ser empregado [5].

A inserção da energia eólica em ambientes urbanos para geração de energia elétrica é considerada uma importante fonte alternativa para o fornecimento da energia elétrica ao sistema próximo ás áreas de consumo, contribuindo para a redução dos impactos ambientais em decorrência da diminuição da emissão de $\mathrm{CO}_{2}$ na atmosfera. A conexão de aerogeradores de pequeno porte em ambientes urbanos apresenta custos relacionados ao investimento inicial com a compra e instalação, operação, manutenção, desenvolvimento e planejamento do projeto, tempo de vida da instalação e custo da produção da energia competindo com o custo da energia tradicional fornecida pela concessionária, porém deve ser observado que a aceitação social leva em consideração um grande número de fatores, tais como, o púbico interessado, as vantagens e interesses na utilização por parte de consumidores donos de empresas, desenvolvedores de projetos, posto que, aderir a esta forma de geração pode contribuir em aspectos como bem estar social, qualidade de vida, melhorar a visibilidade da empresa por parte da sociedade, beneficiar o ecossistema, desenvolver políticas e estratégias, e avaliar com detalhes a separação de custos e benefícios decorrente da implantação da tecnologia [6].

A Alemanha através do desenvolvimento de uma política industrial verde, em que o governo intervém na reestruturação econômica do país em busca de um meio ambiente sustentável a partir da transição energética, iniciou um processo de minimização dos impactos ambientais a partir do desenvolvimento de sua indústria com aplicação inovadora de tecnologias de energias renováveis. Nesta política são comparadas a energia solar fotovoltaica e a eólica, tendo a competitividade, estimulo à inovação, criação de empregos, alterações climáticas, e redução dos custos para os consumidores, como objetivos principais para a transformação econômica com base nesta política industrial. $O$ estudo da política energética de um país está vinculado ao custo de sua energia, seja no consumo industrial ou residência, logo, este mostra-se como um importante critério para avaliar o desenvolvimento e a perspectiva da utilização de energias renováveis para a implementação de um processo de transição energética [7].

\section{PROJETO CIDADE INTELIGENTE BÚZIOS}

O projeto Cidade Inteligente Búzios está sendo implantado em Búzios no estado do Rio de Janeiro, e tem como objetivo o desenvolvimento de um projeto piloto para estudo da implantação de pequenas unidades de geração próximas aos consumidores utilizando a energia solar através de sistemas de painéis fotovoltaicos, e eólica com a geração de eletricidade através de pequenos aerogeradores. Está previsto o atendimento de 10.363 clientes, sendo 13 industriais, 1.518 instalações comerciais/ Serviços públicos e 8.832 unidades residenciais, desenvolvendo também um programa de instalação de 25 pontos de distribuição com interruptor automático, e o uso de 3 linhas de média tensão (15 kV), apresentando $67 \mathrm{~km}$ de extensão, 450 transformadores de média e baixa tensão, e com previsão de consumo de energia de $55 \mathrm{GWh} /$ ano [8].

Estão sendo instalados 12 módulos de geração distribuída com potência de 1,5 e 5 $\mathrm{kWp}$ em diferentes unidades consumidoras, sendo $65 \mathrm{~kW}$ a potência instalada inicialmente, no qual, $45 \mathrm{kWp}$ será de geração solar fotovoltaica, $5 \mathrm{~kW}$ em sistemas de armazenamento através de baterias e $15 \mathrm{~kW}$ em geração eólica, onde o tipo de geração é definido de acordo com a fonte de energia disponível no local, e a 
potência do projeto estará de acordo com o imóvel ao qual será instalado, suprindo parcialmente ou na integra a máxima demanda da unidade consumidora [9].

A seguir serão apresentados alguns dos sistemas instalados pelo Projeto Cidade Inteligente Búzios.

\subsection{Sistemas de Geração Fotovoltaico e Eólico}

Alguns exemplos de sistemas implantados no projeto são: geração solar instalado no Centro de Monitoramento da Ampla e constituído por um sistema fotovoltaico com 40 painéis de filme fino com potência de $130 \mathrm{Wp}$ cada, Figura 1.

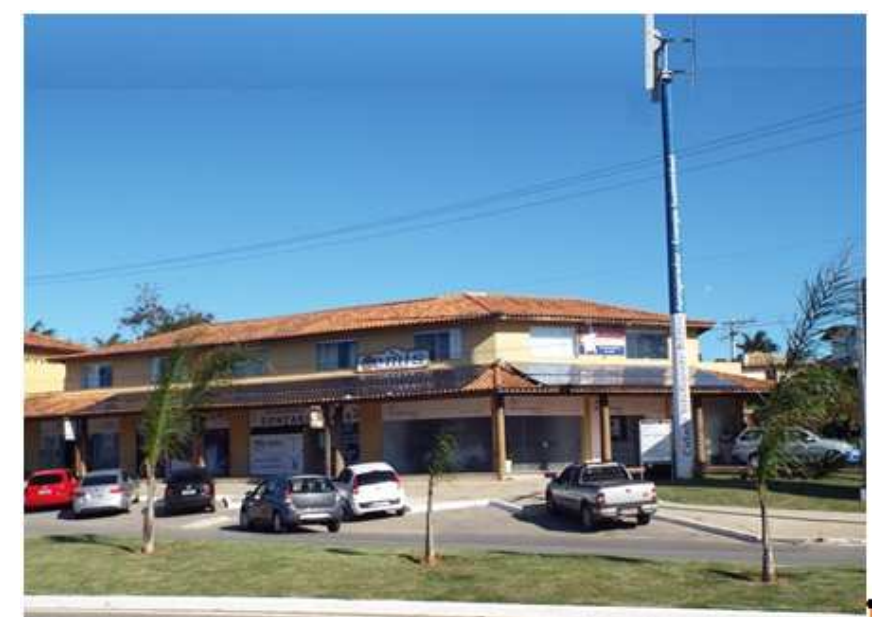

Figura 1. Instalação do Sistema Fotovoltaico e Eólico [9].

A APAE-Búzios apresenta 18 painéis de material policristalino com potência de 245 Wp por unidade instalados, podendo ser observado pela Figura 2.

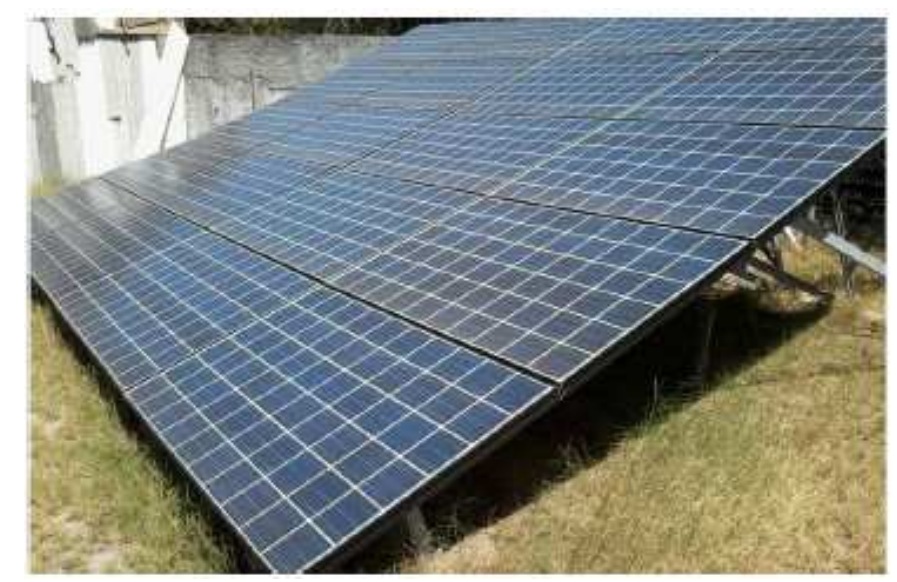

Figura 2. Instalação do Sistema Fotovoltaico Policristalino na APAE-Búzios.

Seguindo a linha de sistemas fotovoltaicos têm-se 20 painéis de material policristalino com potência de 245 Wp no Colégio Municipal Paulo Freire, Figura 3, e no Clube de Tênis, Figura 4. 


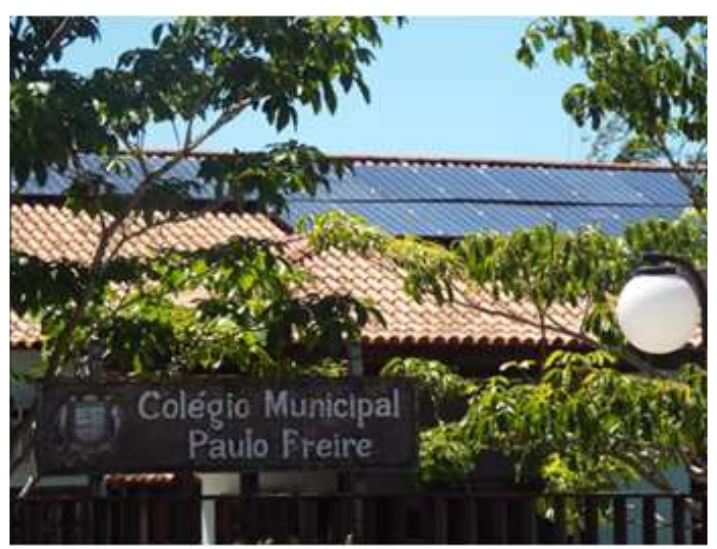

Figura 3. Instalação do Sistema Fotovoltaico no Colégio Municipal Paulo Freire [9].

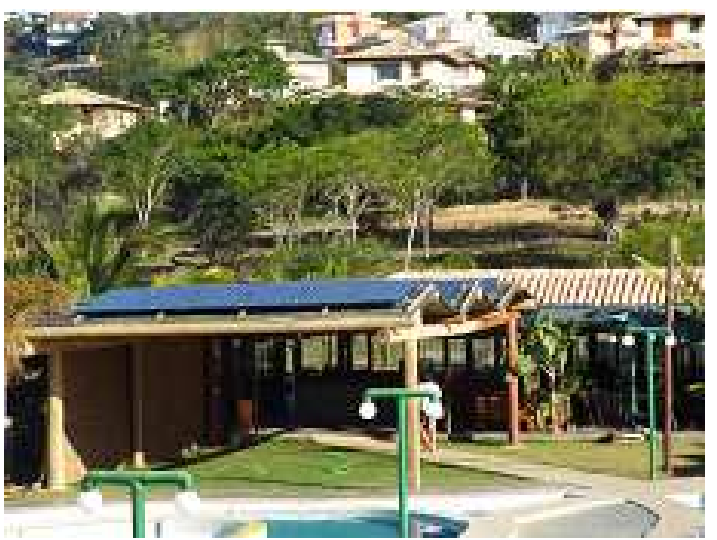

Figura 4. Instalação do Sistema Fotovoltaico no Clube de Tênis [9].

O sistema de geração eólico é composto por um aerogerador de eixo vertical com potência de $1500 \mathrm{~W}$ localizado no Centro de Monitoramento da Ampla, e três de mesma potência e tipo, no Porto da Barra, Figura 1 e Figura 5, respectivamente.

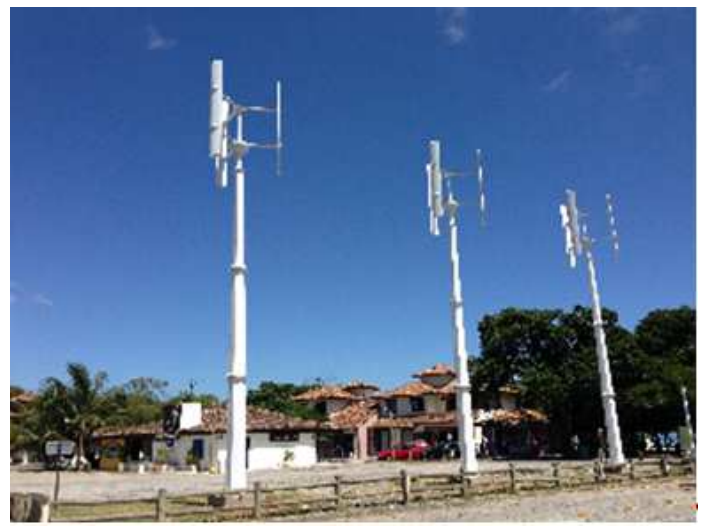

Figura 5. Instalação dos Aerogeradores de Eixo Vertical no Porto da Barra [9].

\section{RESULTADOS E DISCUSSÃO}

Entre vários resultados que o projeto pode fornecer para a indústria, academia e sociedade sobre o comportamento destas novas fontes conectadas a rede de distribuição da concessionária, destacamos:

a) Avaliação da distorção harmônica de corrente gerado pelo inversor do sistema fotovoltaico. A Tabela 1 apresenta um quadro resumo de uma das fases. 
Tabela 1. Conteúdo Harmônico da Corrente - Fase R

\begin{tabular}{|c|c|c|}
\hline Ordem do Harmônico & Corrente na Fase R (A) & Corrente na Fase R (\%) \\
\hline 1 & 15,81 & 100 \\
\hline 2 & 0,541 & 3,42 \\
\hline 3 & 0,263 & 1,66 \\
\hline 4 & 0,287 & 1,81 \\
\hline 5 & 0,287 & 1,81 \\
\hline 6 & 0,081 & 0,51 \\
\hline 9 & 0,115 & 0,72 \\
\hline 11 & 0,158 & 0,99 \\
\hline 13 & 0,163 & 1,02 \\
\hline 15 & 0,072 & 0,45 \\
\hline 19 & 0,110 & 0,69 \\
\hline & 0,081 & 0,51 \\
\hline
\end{tabular}

b) Avaliação da distorção harmônica de tensão do sistema gerador fotovoltaico (após o inversor) e comparação de dados com normas internacionais vigentes. Ver Tabelas 2 e 3.

Tabela 2. Conteúdo Harmônico de Tensão - Fase R

\begin{tabular}{|c|c|c|}
\hline Ordem do Harmônico & Tensão da Fase R-Neutro (V) & Tensão da Fase R-Neutro (\%) \\
\hline 1 & 123,18 & 100 \\
\hline 3 & 0,441 & 0,35 \\
\hline 11 & 0,522 & 0,42 \\
\hline
\end{tabular}

Tabela 3 - Valores de Referência de Distorção Harmônica Totais em percentual da Tensão Fundamental [10]

\begin{tabular}{|c|c|}
\hline Tensão Nominal do Barramento & Distorção Harmônica Total de Tensão (\%) \\
\hline $\mathrm{V}_{\mathrm{N}} \leq 1 \mathrm{kV}$ & 10 \\
\hline $1 \mathrm{kV} \leq \mathrm{V}_{\mathrm{N}} \leq 13,8 \mathrm{kV}$ & 8 \\
\hline $13,8 \mathrm{kV} \leq \mathrm{V}_{N} \leq 69 \mathrm{kV}$ & 6 \\
\hline $69 \mathrm{kV} \leq \mathrm{V}_{\mathrm{N}} \leq 230 \mathrm{kV}$ & 3 \\
\hline
\end{tabular}

c) Avaliação de espectros harmônicos de sistemas aerogeradores e fotovoltaicos, para analises com conceitos de qualidade de energia. As Figuras 6 e 7 apresentam exemplos de resultados obtidos em uma das fases de um aerogerador eixo vertical e sistema fotovoltaico do centro de monitoramento, respectivamente.

d) Análise da redução de perdas não técnicas com estudos sobre o melhor posicionamento das fontes de geração distribuída frente aos consumidores.

Considerando aspectos não relacionados a aplicação das fontes, pode-se citar como pontos importantes e desenvolvidos neste projeto:

e) Conscientização da sociedade local sobre o uso de fontes renováveis como alternativa aos combustíveis fosseis. Existem campanhas locais na comunidade de Buzios abordando este tópico.

f) Aplicação de medidores inteligentes e avaliação de novas opções tarifárias, com tarifas variáveis com o horário de consumo.

g) Divulgação de projetos relacionados a mobilidade com: carro elétrico, bicicleta elétrica e barco solar;

h) Automação de sistemas de distribuição, como operação remota e automática de religadores e acompanhamento de curva de carga de alimentadores. 
i) Aplicação na iluminação publica de luminárias com tecnologia led, sendo alimentadas por painéis fotovoltaicos.

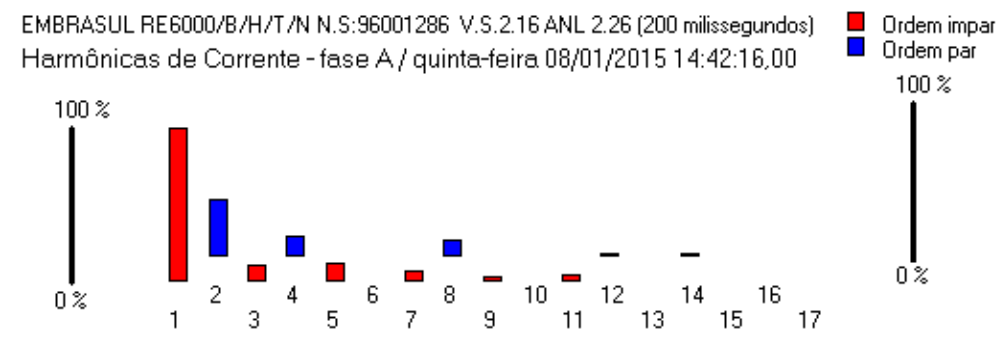

Figura 6. Espectro de corrente da fase $\mathrm{R}$ na saída do inversor de um aerogerador eixo vertical

EMBRASUL RE6000/B/H/T/N N.5:96001206 V.5.2.16.ANL 2.26 [200 milissegundos]

Sinal de Corrente - fase A/ quinta-feira 08/01/2015 14:42:16.00

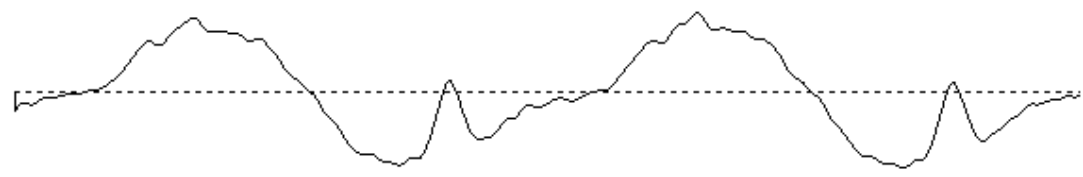

Figura 7. Espectro de corrente da fase R na saída do inversor do sistema fotovoltaico do centro de monitoramento

\section{CONCLUSÃo}

Todos os setores da economia buscam alternativas para reduzir a "conta energia" e entre as opções se destaca a instalação de novas tecnologias como pequenas centrais geradoras solares e/ou eólicas.

Projetos como o Smart City Búzios são importantes para avaliação das novas tecnologias de geração de energia conectadas as redes de distribuição. Neste modelo de projeto, equipamentos de diferentes fabricantes são instalados e avaliados em cenários de carregamento e conexões diferentes dos teóricos.

Entende-se que esta atividade é complementar aos ensaios realizados em laboratórios e apoiam os fabricantes a desenvolverem novas soluções para atender questões levantadas pelo instalador, concessionária, academia e clientes. Além do foco de desenvolvimento da tecnologia, existe o aspecto relacionado ao desenvolvimento de novos profissionais para trabalhar com a tecnologia, atividade esta realizada em parceria com universidades e outros centros de formação profissional.

O aspecto divulgação a sociedade das energias renováveis deve também ser citado, visto que pequenos projetos dentro da cidade com turismo intensivo torna a visibilidade e discussão das tecnologias um tema recorrente. Os exemplos e dados coletados para instalações de pequeno porte (como neste projeto) podem ser expandidas para implantação de geradores similares em pequenas industrias, centros comerciais, edificações, sistemas tratamento de água isolados e outros.

Entre outros dados obtidos, pode-se avaliar o impacto destas novas fontes a rede de distribuição, a qualidade de energia das novas tecnologias e avaliar possíveis medidas mitigadoras, avaliar o limite de carregamento destas mini-gerações e aspectos relativos a controle automático de potência, proteção elétrica e mecanismos de comunicação remotos.

Destaca-se também neste modelo de projeto o envolvimento da comunidade local através das diversas campanhas de divulgação, pesquisas de opinião, eventos 
técnico-científicos (ex: desafio solar com barcos movidos a energia solar realizado em 2013 e 2014), internet gratuita em alguns pontos da cidade, atividades de divulgação em escolas publicas como gincanas, entre outros.

\section{REFERÊNCIAS}

1 Konzen G.; "Difusão de Sistemas Fotovoltaicos Residenciais conectados à rede no Brasil: Uma Simulação de Bass", Tese de Mestrado, USP, 2014.

2 Ruangpattana S., Klabjan D., Arinez J., Biller S., Optimization of on-site renewable energy generation for industrial sites. IEEE Power Systems Conference and Exposition. 2011:1-8.

3 Halabi M. A., Al-Qattan A., Al- Otaibi A, Application of solar energy in the oil industryCurrent status and future prospects. Renewable and Sustainable Energy Reviews. 2015; 43: 296-314.

4 Boudries R., Khellaf A., Aliane A., Ihaddaden L., Khida F., PV system design for powering na industrial unit for hydrogen production. International Jounal of Hydrogen Energy. 2014; 39(27): 15188-15195.

5 Kalogirou S. A., Tripanagnostopolous Y., Industrial application of PV/T solar energy systems. Applied Thermal Engineering. 2007; 27(8-9): 1259-1270.

6 Ishugah T. F, Li Y., Wang R. Z., Kiplagat J. K. Advances in Wind energy resources exploitation in urban environment: A review. Renewable and Sustainable Energy Reviews. 2014. 37: 613-626.

7 Pegels A., Lutkenhorst W. Is Germany's energy transition a case of successful Green industrial policy? Contrasting Wind and solar PV. Energy Policy. 2014. 74: 522-534

8 Vilaca N. M. C. A. A., Figueiredo V. N., Oliveira L. B., Ferreira V. H., Fortes M. Z., Correira W. L., Pacheco O. L. C. Smart City - Caso de Implantação em Búzios. SODEBRAS. 2014. 9: 16-22.

9 Fortes M. Z., Ferreira V. H., Arariba L. B., Rosa R. S., Correia W. F., Pacheco O. C., Smart City - Experiência para a Expansão de Sistemas de Geração com Fontes Alternativas. IX Congresso Brasileiro de Planejamento Energático - CBPE. 2014: 1-10.

10 ANEEL. Agência Nacional de Energia Elétrica. Procedimento de Distribuição de Energia Elétrica no Sistema Elétrico Nacional - PRODIST Módulo 8 - Qualidade de Energia Elétrica. Acesso em 01 de Janeiro de 2015. 